Association for Information Systems

AIS Electronic Library (AISeL)

Wirtschaftsinformatik Proceedings 2005

Wirtschaftsinformatik

February 2005

\title{
Standardisierte Mikrosimulation eines Kapitalmarktes mit scale-free Netzwerken und Informationsansteckung
}

Oliver Hein

Johann Wolfgang Goethe-Universität Frankfurt

Michael Schwind

Johann Wolfgang Goethe-Universität Frankfurt

Follow this and additional works at: http://aisel.aisnet.org/wi2005

\section{Recommended Citation}

Hein, Oliver and Schwind, Michael, "Standardisierte Mikrosimulation eines Kapitalmarktes mit scale-free Netzwerken und Informationsansteckung" (2005). Wirtschaftsinformatik Proceedings 2005. 86.

http://aisel.aisnet.org/wi2005/86

This material is brought to you by the Wirtschaftsinformatik at AIS Electronic Library (AISeL). It has been accepted for inclusion in Wirtschaftsinformatik Proceedings 2005 by an authorized administrator of AIS Electronic Library (AISeL). For more information, please contact elibrary@aisnet.org. 
In: Ferstl, Otto K, u.a. (Hg) 2005. Wirtschaftsinformatik 2005: eEconomy, eGovernment, eSociety; 7. Internationale Tagung Wirtschaftsinformatik 2005. Heidelberg: Physica-Verlag

ISBN: 3-7908-1574-8

(C) Physica-Verlag Heidelberg 2005 


\title{
Standardisierte Mikrosimulation eines Kapitalmarktes mit scale-free Netzwerken und Informationsansteckung
}

\author{
Oliver Hein, Michael Schwind \\ Johann Wolfgang Goethe-Universität Frankfurt
}

Zusammenfassung: Die Mikrosimulation von Finanzmärkten hat in den letzten Jahren große Fortschritte erzielt. Die wichtigsten stilisierten Fakten der Finanzmärkte konnten bereits mit Hilfe von Multi-Agenten-Modellen nachvollzogen werden. Die Implementationen der verwendeten Modelle sind allerdings immer noch sehr heterogen und zumeist kaum nachvollziehbar. Eine gemeinsame Plattform zur Entwicklung und zum Vergleich von Marktmodellen wäre wünschenswert. Der FASM (Frankfurt Artificial Stock Market) stellt eine solche Plattform dar. Der FASM wurde unter Java auf Basis der FIPA konformen Middleware Jade entwickelt und erfüllt damit einen wichtigen Industriestandard. Der FASM verfügt darüber hinaus über die Möglichkeit Preisbildungsprozesse mit Hilfe von Netzwerkstrukturen wie scale-free-Netzwerken und Informationsdiffusionsprozessen erforschbar zu machen.

Schlüsselworte: Mikrosimulation, Multi-Agenten-Modelle, Finanzmärkte, Jade, scale-free

\section{Einführung}

In die Prognose von Finanz- und Warenmärkten werden jedes Jahr weltweit große Summen investiert, die Erfolge sind bisher zumeist eher mäßig bis ungenügend [Spi02; Spi03; Spi04]. Fehlprognosen verursachen hohe Schäden bei Banken und Versicherungen und stellen ein großes Problem für die Altersversorgung dar. Woran liegt der mangelnde Erfolg der Analysten? Die Effizienzhypothese der Kapitalmärkte stellt Anforderungen an die Märkte, die vor allem in Phasen erhöhter Unsicherheit oft nicht haltbar sind. Was verursacht die Abweichungen?

Die endgültigen Antworten auf diese Fragen sind in nächster Zeit nicht zu erwarten, dennoch gibt es neuere Forschungsansätze, die bisher vernachlässigte Aspekte des wirtschaftlichen Handelns genauer untersuchen. Preisbildungsprozesse scheinen über endogene Eigenschaften zu verfügen, die unter bestimmten Voraussetzungen zu erheblichen Abweichungen von klassischen Bewertungsmaßstäben bei- 
tragen. Die dynamische Interaktion zwischen Marktteilnehmern, wie das Herdenverhalten, in Verbindung mit steigendem Einfluss von trendorientierten Investoren wie z.B. Charttechnikern und Momentumtradern, scheint ein Grund für immer wieder auftretende Kapitalmarktanomalien - wie z.B. Booms und Crashes - zu sein. Modelle zur Mikrosimulation von Finanzmärkten können zur Untersuchung dieser Phänomene beitragen. Ob diese Modelle auch zur Prognose oder vielleicht zu einer erfolgreichen Asset-Allocation beitragen können, ist heute noch nicht absehbar [Far01].

Die vorliegende Arbeit beschreibt einen neuen Modellansatz, der sich einerseits durch seinen standardisierten Aufbau als Plattform für Experimente mit Kapitalmarktsimulationen auszeichnet und andererseits einen Schwerpunkt innerhalb der Inter-Agenten-Kommunikation setzt, der bei Vorgängermodellen nur implizit vertreten ist. Es besteht somit erstmals die Möglichkeit die Topologie eines Kommunikationsnetzes und die Dynamik von Ansteckungsprozessen in ihrem Verhältnis zur Preisbildung an Kapitalmärkten zu untersuchen.

\section{Mikrosimulation von Kapitalmärkten}

Zur Erörterung des Forschungsgebietes „Mikrosimulation von Kapitalmärkten“ werden im folgenden typische Simulationsmodelle vorgestellt. Die Validierung der Modelle erfolgt in der Regel durch Nachweis der empirisch feststellbaren universellen stilisierten Fakten von Finanzmärkten.

\subsection{Simulationsmodelle für Kapitalmärkte}

Die Erforschung der komplexen Dynamik von Finanzzeitreihen, wie Aktien- und Wechselkursen, erfährt in jüngster Zeit ein steigendes interdisziplinäres Interesse. Multi-Agenten-Systemen (MAS) kommt in diesem Zusammenhang eine wichtige Rolle zu. Sie eignen sich in besonderer Weise für die Untersuchung des Preisbildungsprozesses an Kapitalmärkten. Das Zusammenspiel einer Vielzahl heterogener, autonom interagierender Agenten spiegelt das tägliche Geschehen an den internationalen Börsenplätzen wider.

Das Auftreten von Anomalien wie Boom- und Crashphasen wird als widersprüchlich zur Effizienzhypothese angesehen, die vom hyperrationalen, nutzenmaximierenden Homo Oekonomicus ausgeht und eine Übereinstimmung vom Preis eines Wertpapiers mit seinen fundamentalen Daten postuliert. Spätestens seit dem Aufkommen des Behavioural Finance hat sich das Bild der Wirtschaftssubjekte geändert [Sim97]. Man geht mittlerweile nur noch von einem begrenzt rationalen Handeln menschlicher Akteure aus. Diese Annahme gibt Raum für neue Verhaltens- 
muster von Investoren aufgrund von Erkenntnissen der experimentellen Ökonomie [KaTv82].

Erst die Mikrosimulation von Finanzmärkten hat einen ersten Einblick in die Interdependenzen eines kollektiven Preisfindungsprozesses ermöglicht. Phänomene wie „self-organizational criticality“ können so besser auf ihre Auswirkung auf die Preisbildung hin untersucht werden. Typisches Anlegerverhalten wie das Herdenverhalten sind modellierbar geworden.

Erste Versuche mit künstlichen Aktienmärkten gehen auf Anfang der neunziger Jahre zurück. Der Artificial Stock Market (ASM) des Santa Fe Institutes hat mit Hilfe von MAS erste Einblicke in die Dynamik von möglichen Preisbildungsprozessen ermöglicht [ $\left.\mathrm{LeB}^{+} 94\right]$. Der ASM lässt seine Investoren ihre Anlagestrategie mit Hilfe von genetischen Algorithmen optimieren. Der ASM wurde mit Hilfe von Swarm ${ }^{1}$, einer Multi-Agenten-Plattform, und der Programmiersprache Objective$\mathrm{C}$ implementiert. Es gibt in diesem Modell keine direkte Agentenkommunikation, allenfalls eine indirekte Kommunikation über die Preisveränderungen.

Es folgten eine ganze Reihe von Modellen, wie das von Levy, Levy, Salomon [ $\left.\mathrm{Lev}^{+} 00\right]$, oder das Modell von Cont, Bouchaud [CoBo00]. Der Genoa Artificial Stock Market (GASM) ist eines der ersten Modelle, das als eine experimentelle Plattform für Kapitalmarktsimulationen dienen soll [ $\left.\mathrm{Rab}^{+} 01\right]$. Es wurde in der Programmiersprache Smalltalk entwickelt und orientiert sich inhaltlich an Cont, Bouchaud. Das Modell von Lux, Marchesi zeigt bereits alle wichtigen stilisierten Fakten, die zur Validierung des Modells dienen [Lux00; LuMa99]. Das Modell von Lux wurde in der Programmiersprache Gauss implementiert.

\subsection{Stilisierte Fakten der Kapitalmärkte}

Die Mikrosimulation von Kapitalmärkten erhält nur dann einen Sinn, wenn sie die Verhältnisse realer Kapitalmärkte angemessen widerspiegelt. Kapitalmärkte verfügen über statistische Eigenschaften, die in verschiedenen Märkten wie Aktien-, Renten-, Devisen-, oder Rohstoffmärkten zu finden sind und als universelle stilisierte Fakten bezeichnet werden [Cont01]. Anhand dieser Eigenschaften können MAS Modelle hinsichtlich ihres Realitätsgehaltes überprüft werden. Die mit den MAS generierten Zeitreihen müssen die universellen stilisierten Fakten ebenso aufweisen wie reale Finanzmarktzeitreihen. Nachfolgend werden die wichtigsten universellen stilisierten Fakten vorgestellt.

\subsubsection{Stationarität und Einheitswurzeln}

Finanzmarktzeitreihen weisen in aller Regel die statistische Eigenschaft der NichtStationarität auf. Ob diese Eigenschaft vorliegt, kann mit Hilfe des Augmented

www.swarm.org 
Dickey-Fuller-Tests (ADF-Test), auch als Einheitswurzel-Test bekannt, überprüft werden. Diesem Test liegt die Vorstellung zugrunde, dass die Veränderungen von Beobachtung zu Beobachtung in der Zeitreihe grundsätzlich wie folgt beschrieben werden kann:

$$
\Delta X_{t}=c+(\alpha-1) X_{t-1}+\sum_{i=1}^{p} \alpha_{i} \Delta X_{t-i}+\varepsilon_{t}
$$

Für $\alpha=1$ (das entspricht $b=0$ mit $b=\alpha-1$ ) gilt, dass ein Random Walk vorliegt, der seinerseits stets als nicht-stationär gilt. Der ADF-Test überprüft nun die Nullhypothese $\mathrm{H}_{0}: \mathrm{b}=0$ (,Random Walk“). Kann die Nullhypothese verworfen werden, wird davon ausgegangen, dass die Hypothese $\left(\mathrm{H}_{1}: \mathrm{b}<0\right)$ gilt, was unter den Annahmen des ADF-Tests auf Stationarität der Zeitreihe schließen lässt [Pod99].

\subsubsection{Fat Tails}

Es hat sich gezeigt, dass die Verteilung logarithmischer Renditen $r_{t}=\ln p_{t}-\ln p_{t-1}$ von Finanzzeitreihen leptokurtisch (Abbildung 1) ist bzw. „fat tails" aufweist und einem Potenzgesetz folgt:

$$
\mathrm{F}\left(\left|\mathrm{r}_{\mathrm{t}}\right|>\mathrm{x}\right) \approx \mathrm{c} \cdot \mathrm{x}^{-\mu}
$$

Dies bedeutet, dass die äußeren Bereiche der Wahrscheinlichkeitsverteilungsfunktion ausgeprägter sind als im Falle der Normalverteilung. Der Exponent $\mu$ fällt gewöhnlicher Weise in das Intervall [2,5]. Der vierte statistische Moment, die Kurtosis, ist bei Finanzzeitreihen $>3$ und zeigt damit eine Abweichung von der Normalverteilung an, die von der Effizienzhypothese gefordert wird. 


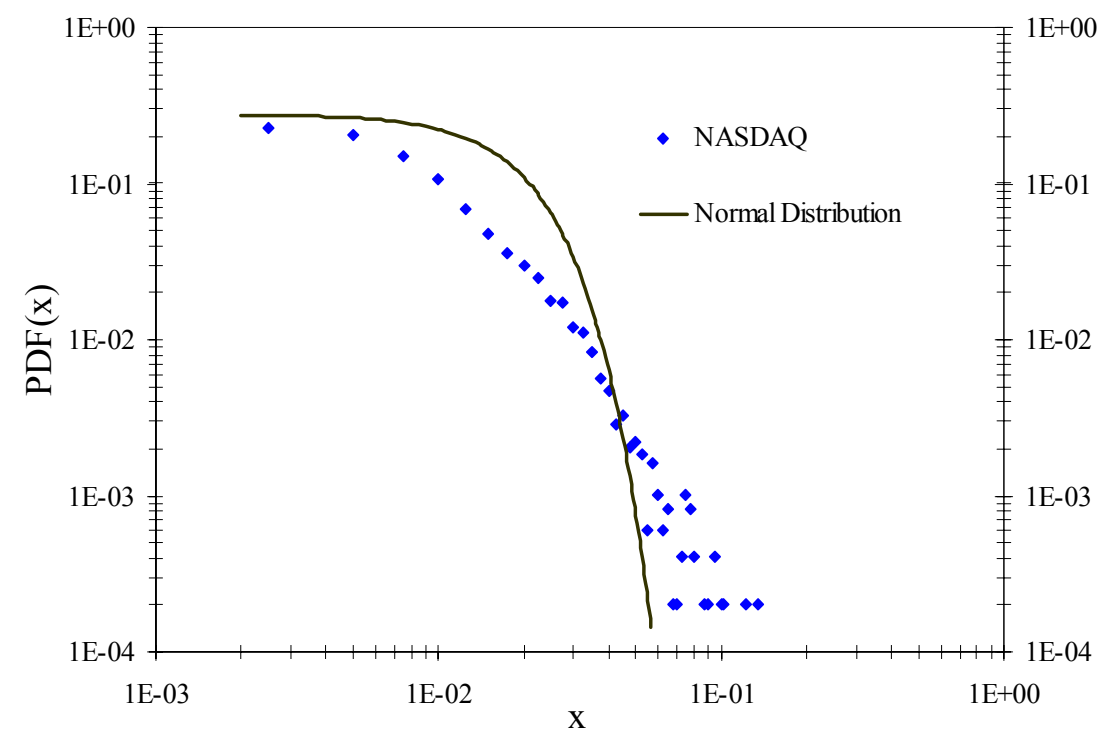

Abbildung 1: Log-Log-Plot der absoluten Wahrscheinlichkeitsverteilungsfunktion PDF(x) des NASDAQ Index auf Tagesbasis vom 13.11.1984 bis 18.05.2004 im Vergleich zur Normalverteilung mit gleichem Durchschnitt und gleicher Standardabweichung.

\subsubsection{Volatility Clustering}

Finanzzeitreihen verfügen nachweisbar über keine lineare Autokorrelation, was als Unterstützung der Effizienzmarkthypothese angesehen wird [Con99]. Würde es eine signifikante Autokorrelation geben, könnte man Arbitragegewinne erzielen. Es hat sich allerdings gezeigt, dass die Abwesenheit von Autokorrelation nicht für die Volatilität von Wertpapierkursen gilt. Abbildung 2 zeigt eine erhöhte Autokorrelation bei den beiden Volatilitätsmassen absolute und quadratische Returns im Vergleich zu einfachen Returns an. Hohe (niedrige) Volatilität in der Gegenwart ist daher ein guter Indikator für hohe (niedrige) Volatilität in der näheren Zukunft. Es besteht außerdem noch eine Korrelation mit dem erzielten Volumen an Transaktionen. An volatilen Handelstagen werden in der Regel auch höhere Umsätze erzielt [Con01]. 

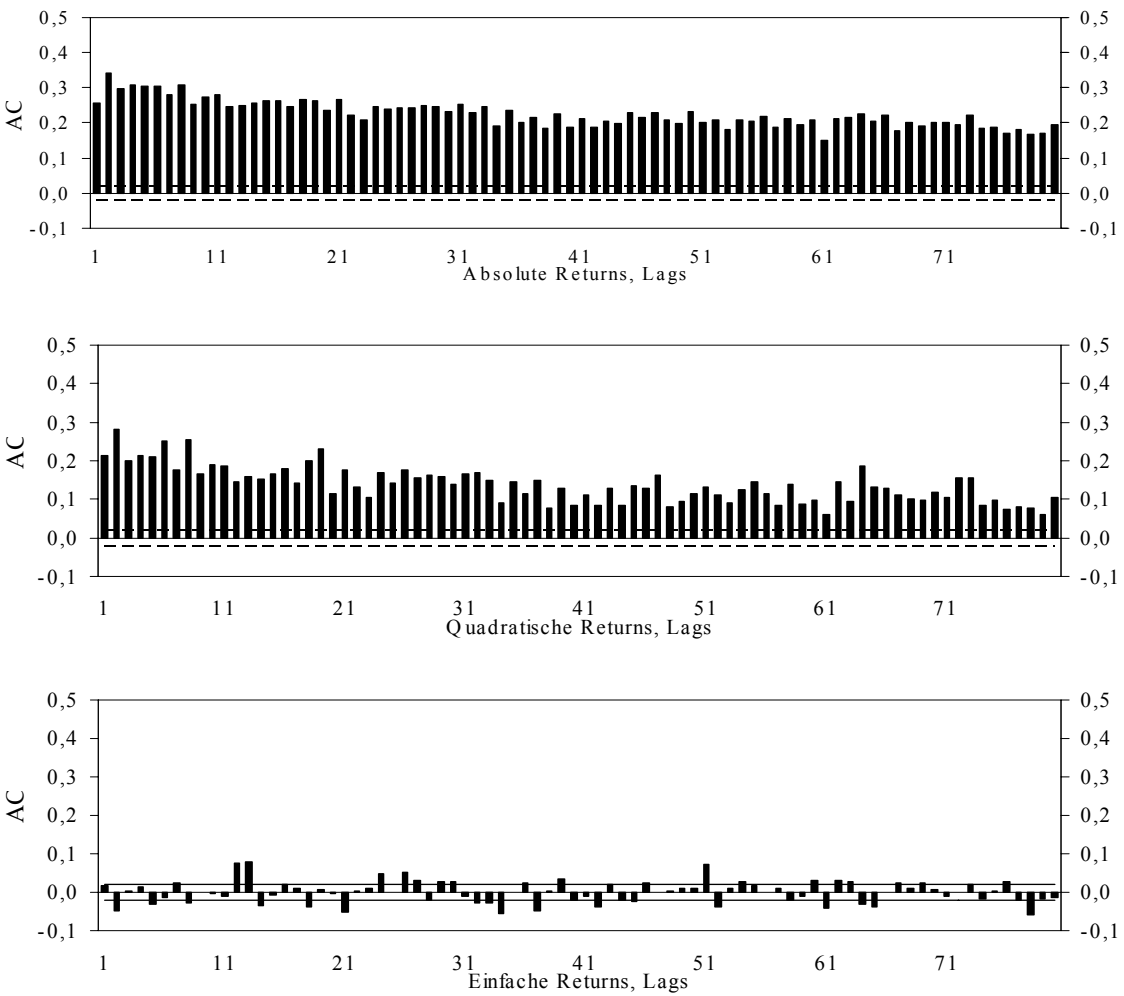

Abbildung 2: Autokorrelation AC für die Volatilitätsmasse absolute Returns und quadratische Returns im Vergleich zu einfachen Returns des NASDAQ Index vom 13.11.1984 bis 18.05.2004 bei verschiedenen Lags.

\section{FASM - Frankfurt Artificial Stock Market}

Die Entwicklung eines weiteren Simulationsmodells für Kapitalmärkte ist aus der Notwendigkeit der besseren Nachvollziehbarkeit von erzielten Ergebnissen in einer standardisierten Umgebung einerseits und der besseren Erforschung der zugrunde liegenden Organisationsstruktur und Entwicklungsdynamik von Agentenpools andererseits entstanden. Frankfurt als Sitz der Johann-Wolfgang-vonGoethe-Universität mit Forschungsschwerpunkt Wirtschaftsinformatik und Finanzen innerhalb des Fachbereichs Wirtschaftswissenschaften, hat sich für das Modell als Namensgeber angeboten. Der FASM soll durch seinen modularen Aufbau als Entwicklungsplattform für experimentelle Märkte dienen und durch seine Stan- 
dardisierung durch Verwendung von $\mathrm{Jade}^{2} 3.2$ den Austausch mit anderen Forschungsgruppen ermöglichen.

Im Folgenden werden die wesentlichen Komponenten dieses neuen experimentellen Marktes beschrieben.

\subsection{Die Agenten}

Der Agentenpool teilt sich in zwei grundlegende Typen: Fundamentalinvestoren (FI) und Trendinvestoren (TI) [FaSh02].

FI orientieren sich am inneren Wert eines Wertpapiers. Der innere Wert $v_{t}$ ist exogen vorgegeben und folgt einem random walk:

$$
\mathrm{v}_{\mathrm{t}+1}=\mathrm{v}_{\mathrm{t}}+\eta_{\mathrm{t}+1}
$$

mit $\eta_{\mathrm{t}}$ als normalem IID (independently and identically distributed) Zufallsprozess mit Standardabweichung $\delta_{\eta}$ und Durchschnitt $\mu_{\eta}$. Eine einfache Fundamentalstrategie für den inneren Wert $v_{t}$ und die Position an Wertpapieren $x_{t}$ hat die Form:

$$
x_{t+1}=x\left(v_{t}, p_{t}\right)=V\left(v_{t}-p_{t}\right)
$$

mit $\mathrm{V}$ ansteigend und $\mathrm{V}(0)=0$. Abhängig von der Fehlbewertung $\mathrm{m}_{\mathrm{t}}=\mathrm{p}_{\mathrm{t}}-\mathrm{v}_{\mathrm{t}}$ werden Käufe bei Unterbewertung und Verkäufe bei Überbewertung ausgelöst.

TI beobachten den Preisverlauf und versuchen einem Trend zu folgen, die Strategie der TI stellt sich folgendermaßen dar: für eine Zeitskala $\theta$ heißt eine Tradingstrategie trendorientiert, wenn die Position an Wertpapieren $\mathrm{x}_{\mathrm{t}}$ eine positive Korrelation $\rho$ mit vergangenem Preis $p_{t}$ hat:

$$
\rho\left(\mathrm{x}_{\mathrm{t}+1},\left(\mathrm{p}_{\mathrm{t}}-\mathrm{p}_{\mathrm{t}-\theta}\right)\right)>0 .
$$

Beide Gruppen starten mit einem vorgegebenen Startvermögen an Liquidität und Wertpapieren. Ein Agent kann seine Handelsstrategie wechseln, wenn die eigene Strategie im Vergleich zu anderen Agenten in seinem Netzwerk weniger erfolgreich ist. Dazu ist es notwendig Schwellenwerte zu definieren, die zwischen den Agenten variieren können und eine Kommunikationsstruktur, die einen Wechsel der Handelsstrategie aufgrund eines Herdenverhaltens bewirken.

www.jade.cselt.it 


\subsection{Die Topologie des Agentennetzwerks}

Durch Watts, Strogatz [WaSt98] wurde 1998 die „small world“-Eigenschaft für Netzwerke eingeführt und ihre Ähnlichkeit mit in der Natur auftretenden Netzwerken gezeigt. Barabasi, Alberts weiterführende Arbeit von 1999 zeigte die Eigenschaften von scale-free Netzwerken und Übereinstimmungen mit natürlichen Netzwerken auf [BaA199]. Für eine möglichst realistische Topologie der Agentenkommunikation sollen innerhalb des Models Kommunikationsstrukturen verwendet werden, die auch innerhalb sozialer Netze anzutreffen sind. Im Folgenden werden die wesentlichen Charaktere von scale-free-Netzwerken erläutert.

Netzwerke können durch die folgenden drei Parameter gekennzeichnet werden [WaCh03]:

1. Durchschnittliche Pfadlänge:

Wenn die Distanz $\mathrm{d}_{\mathrm{ij}}$ die minimale Anzahl von Kanten zwischen Knoten i und

$\mathrm{j}$ ist, dann ist die durchschnittliche Pfadlänge der Durchschnitt der minimalen Distanzen zwischen allen Knoten.

2. Clustering Koeffizient:

Angenommen Knoten $\mathrm{i}$ hat $\mathrm{k}_{\mathrm{i}}$ Kanten und ist damit mit $\mathrm{k}_{\mathrm{i}}$ Knoten verbunden. Zwischen diesen Knoten kann es maximal $\mathrm{k}_{\mathrm{i}}\left(\mathrm{k}_{\mathrm{i}}-1\right) / 2$ Kanten geben, wenn alle Knoten miteinander verbunden sind. Der Clustering Koeffizient $C_{i}$ des Knoten $\mathrm{i}$ ist dann definiert als der Quotient der existierenden Kanten $\mathrm{E}_{\mathrm{i}} \mathrm{zu}$ den maximal möglichen Kanten:

$\mathrm{C}_{\mathrm{i}}=2 \mathrm{E}_{\mathrm{i}} /\left(\mathrm{k}_{\mathrm{i}}\left(\mathrm{k}_{\mathrm{i}}-1\right)\right)$

Der Clustering Koeffizient des Netzwerkes ist der Durchschnitt über alle $C_{i}$.

3. Gradverteilung:

Der Grad eines Knoten ist definiert als die Anzahl seiner Kanten. Die Verteilung der Grade der Knoten eines Netzwerkes kann durch eine Verteilungsfunktion $\mathrm{P}(\mathrm{k})$ charakterisiert werden, die angibt wie hoch die Wahrscheinlichkeit ist, dass ein Knoten den Grad k hat. Innerhalb regulärer Netzwerke besitzen alle Knoten den gleichen Grad k, P(k) hat dadurch lediglich eine Spitze bei k. Bei zufälligen Netzwerken wird die Form von $\mathrm{P}(\mathrm{k})$ um den Maximalwert der Funktion herum verbreitert und folgt einer Poisson-Verteilung. Die PoissonVerteilung fällt exponentiell vom Maximalwert ab. Es konnte empirisch festgestellt werden [WaCh03], dass $\mathrm{P}(\mathrm{k})$ von realen komplexen Netzwerken, wie Fluglinien-Netzen, Sprache, Internet domains, Autoren-Datenbanken, u.a., nicht zu den Poisson-Verteilungen gehört, sondern nur einen Abfall vom Maximalwert zeigt der einem Potenzgesetz folgt: 


$$
\mathrm{P}(\mathrm{k}) \square \mathrm{k}^{-\gamma}
$$

Scale-free-Netzwerke haben ihren Namen ihrer Eigenschaft zu verdanken, dass Potenzgesetze frei von charakteristischen Skalen sind. Durch den langsameren Abfall vom Maximalwert existieren Knoten mit einem höheren Grad als der Durchschnitt der Knoten, so genannte Superknoten. Beim Blick auf ein Flugliniennetz fallen diese Superknoten als die Hauptumsteigepunkte, wie der Frankfurter Flughafen, sofort auf. Abbildung 3 zeigt ein scale-free-Netzwerk mit 25 Knoten, einer durchschnittlichen Pfadlänge von 2,14 und einem Clustering Koeffizient von 0,328 .

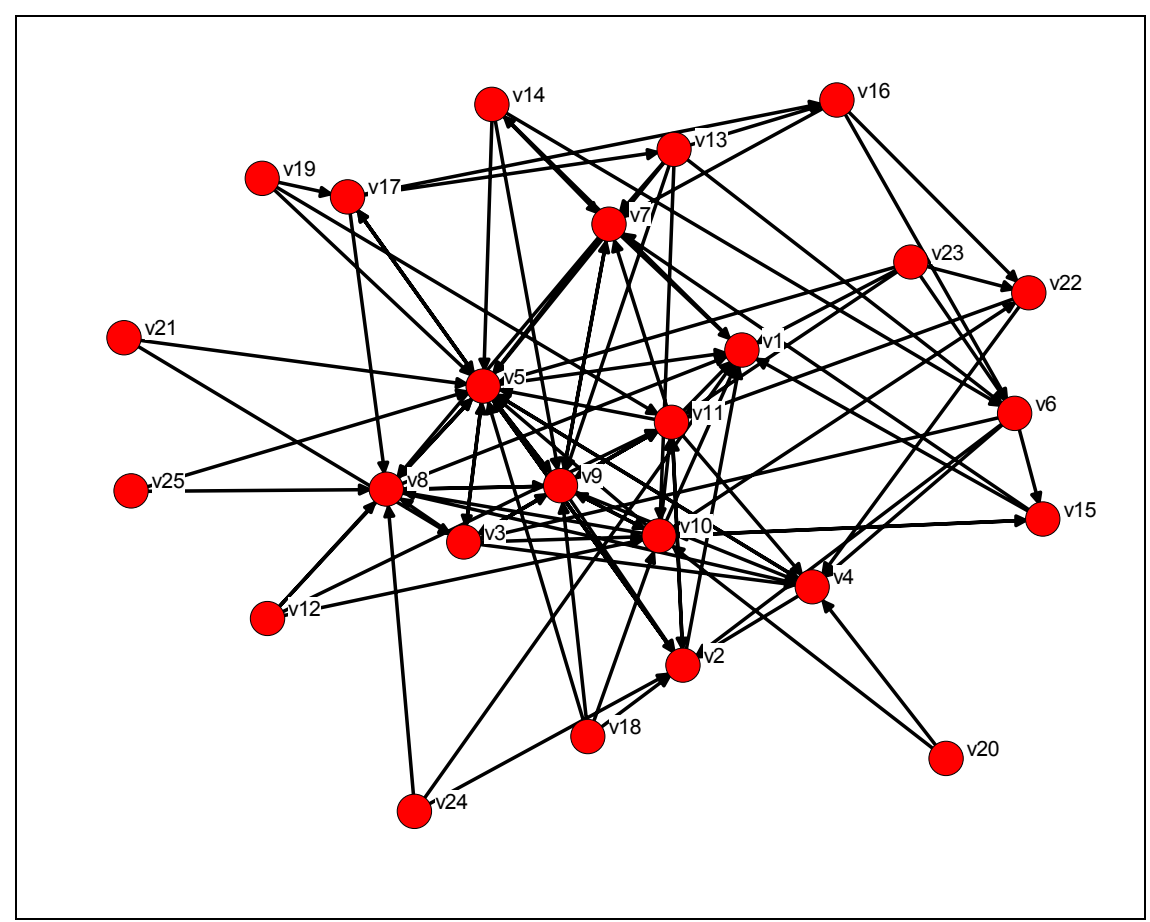

Abbildung 3: Beispiel für ein scale-free Agenten Netzwerk

Die Generierung eines scale-free-Netzwerkes ist durch Barabasi/Albert folgendermaßen definiert worden [BaBo03]:

1. Wachstum: Starte mit einer kleinen Anzahl $\mathrm{m}_{0}$ von Knoten. Füge in jedem Schritt einen neuen Knoten ein und verbinden ihn mit $\mathrm{m} \leq \mathrm{m}_{0}$ bereits existierenden Knoten. 
2. Bevorzugtes Verbinden: Die Wahrscheinlichkeit $\Pi_{i}$, dass ein neuer Knoten mit Knoten i verbunden wird, hängt vom Grad des Knoten i folgendermaßen ab:

$$
\Pi_{\mathrm{i}}=\mathrm{k}_{\mathrm{i}} / \sum_{\mathrm{j}} \mathrm{k}_{\mathrm{j}}
$$

Übertragen auf die Organisationsstruktur von Wertpapierinvestoren, können von Medien (Börsen-TV, Zeitungen, Aktienbriefe), Kapitalsammelstellen und Vermögensberatern (Banken, Versicherungen, Fonds) und einflussreiche Individuen (George Soros) als Superknoten modelliert werden. Sie bestimmen aufgrund ihres höheren Einflusses einen wesentlichen Teil der Marktmeinung.

Die Topologie eines Netzwerkes hat wesentlichen Einfluss auf die dynamischen Prozesse, die sich in ihm abspielen. Der Mechanismus der Diffusion von Informationen, die einen großen Einfluss auf die Entscheidungsfindung eines Investors ausübt, muss als nächstes definiert werden.

\subsection{Informationsansteckung}

Die epidemische Ansteckung in komplexen Netzwerken hat viele Beispiele in realen Netzen. Die Erforschung der Ausbreitung von Krankheiten wie AIDS und Ebola, von Computerviren im Internet, von kaskadenartigen Ausfällen in Energienetzen, oder Moden in sozialen Netzen, haben gemeinsame Wurzeln und können mit Hilfe von Netzwerken untersucht werden. Das Standard SIS-Model (susceptible-infected-susceptible) wird zur Modellierung des Ansteckungsprozesses verwendet [PaVe01]. Knoten repräsentieren Agenten, die entweder im Zustand „empfänglich“ oder ,infiziert" sein können und über Kanten mit anderen Agenten verbunden sind. Ein infizierter Agent kann einen verbundenen Agenten mit Wahrscheinlichkeit $v$ anstecken, gleichzeitig kann ein infizierter Agent mit Wahrscheinlichkeit $\sigma$ vom Zustand „infiziert“ in den Zustand „empfänglich“ zurück wechseln. Die effektive Verbreitung $\lambda$ ergibt sich durch:

$$
\lambda=v / \delta
$$

Für homogene Netzwerke, wie small-world-Netzwerke und Zufallsnetze, gilt die Existenz einer Ausbreitungsrate $\lambda_{\mathrm{c}}>0$. Ist $\lambda \geq \lambda_{\mathrm{c}}$ bleibt die Infektion im Netzwerk erhalten, bei $\lambda<\lambda_{c}$ verschwindet die Infektion. Für scale-free-Netzwerke dagegen liegt $\lambda_{c}$ nahe Null, eine Infektion breitet sich schnell und über weite Bereiche des Netzwerkes aus und behält hohe Beständigkeit.

Übertragen auf die Modellbeschreibung eines künstlichen Aktienmarktes kann eine schnelle und weitreichende Diffusion von Information in Form von Gerüchten in Kombination mit begrenzt rationalem Handeln der Marktteilnehmer eine Ab- 
weichung des Marktpreises vom Fundamentalwert bewirken. Im Modell wird dieser Mechanismus zur Implementierung der Strategiewechsel zwischen fundamentaler und trendorientierter Handelsstrategien der Agenten benutzt.

\subsection{Der Auktionsmechanismus}

Viele der bisher präsentierten Simulationsmodelle für Aktienmärkte verwenden einfache Doppelauktionsmechanismen zur Darstellung des Handelsprozesses an den Börsen. In der Realität wird jedoch meist neben dem unmittelbaren Doppelauktionsmechanismus das Limitorderbuch eingesetzt. Das Limitorderbuch ist die Darstellung aller limitierter Kauf- und Verkaufsorder in Preis und Volumen jeweils in Form einer Warteschlange. Die Orders werden dabei in der aufsteigenden Reihenfolge ihrer Limitpreise geführt. Alle Kauforders liegen unterhalb der „bestens" ausgeführten Kauforder und unterhalb der niedrigsten limitierten Verkaufsorder des Orderbuchs. Eine Transaktion findet statt, wenn neu eintreffende Limitoder Marktorder jeweils über dem Niveau der letzten Verkaufsorder bzw. unter der letzen Kauforder im Limitorderbuch liegen. Bei Limitorders mit dem gleichen Limitpreis werden die zuerst eingetroffenen Orders bevorzugt. Beim Matching wird dabei die temporäre Teilerfüllung von Aufträgen in Kauf genommen, bis die Order ganz erfüllt ist $\left[\mathrm{Rab}^{+} 03\right]$. Die Bedeutung der Einführung des Limitorderbuchs für unser Modell hängt mit seinem nachgewiesenen Einfluss auf den Preisbildungsprozess zusammen [Smi $\left.{ }^{+} 03\right]$. Der Einsatz des Limitorderbuchs führt zur verstärkten Beobachtung von „stylized facts“ [ $\left.\mathrm{Rab}^{+} 03\right]$. So analysieren Biais et al. $\left[\mathrm{Bia}^{+} 95\right]$ den Zusammenhang zwischen dem Orderfluss und dem Orderbuch an der Pariser Börse und kommen zu dem Ergebnis, dass ein hoher Auftragsdurchlauf bei kursnahen Geboten entsteht, während bei etwas weiter vom Kursniveau entfernten Orders, die Tiefe des Auftragsbuchs hoch ist. Bouchaud et al. [Bou ${ }^{+}$2] zeigen auf, dass die eingehenden Limitorders einer relativ breiten exponentiellen Verteilung der Preise folgen. Maslov und Mills diagnostizieren ebenfalls eine für die stilisierten Fakten typische Exponentialverteilung der Limitorder sowie „fat tails“ bei der Preisanalyse der Aufträge im Limitorderbuch [MaMi01]. In einem ökonometrischen Modell untersuchen Lo et al. den Einfluss von Limitpreis, Bid-AskSpread und Marktvolatilität auf die Ausführungszeiten der Aufträge und stellen dabei eine hohe Sensitivität dieser gegenüber dem Limitpreis fest [LoM $\left.{ }^{+} 02\right]$. Aufgrund der hier aufgezeigten Bedeutung des mit der Doppelauktion gekoppelten Limitordermechanismus für die Preisbildung, verwendet der FASM eine Börsenimplementierung mit Limitorderbuch. Dieses Verfahren findet z.B. an der Frankfurter Wertpapierbörse zur Ermittlung der Einheitskurse (Kassakurse) Anwendung. 


\section{Modellimplementation}

Die bisher entwickelten Simulationsmodelle sind genauso vielfältig wie ihre Implementationen. Ergebnisse lassen sich nur schwer nachvollziehen und sind kaum vergleichbar oder gar austauschbar. Eine Standardplattform würde die erzielten Ergebnisse für andere Forscher besser zugänglich machen, das Rad müsste nicht jedes Mal neu erfunden werden. Ausweg bietet das standardisierte MAS Jade 3.2 (Java Agent Development Framework), das die Standards der FIPA ${ }^{3}$ (Foundation for Intelligent Physical Agents) befolgt.

Anders als bei der Simulationsplattform Swarm verfügt Jade über eine ausgeprägte Inter-Agenten-Kommunikation, die für das beschriebene Modell unerlässlich ist und bei dem ASM der unter Swarm entwickelt wurde gänzlich fehlt. Swarm ist außerdem noch in der Programmiersprache Objective-C, einer heute selten benutzen Programmiersprache die ursprünglich für Next Computer entwickelt wurde, implementiert. Swarm ist nicht FIPA konform. Jade als Simulations-Middleware verfügt über alle wichtigen Funktionen, die für eine Kapitalmarktsimulation benötigt werden und ist dadurch sehr flexibel einsetzbar.

Jade vereinfacht die Entwicklung von verteilten Systemen, bestehend aus heterogenen Einheiten, die untereinander kommunizieren und kooperieren [Bel ${ }^{+} 03$ ]. Jade wurde in Java einwickelt und ist durch geeignete API's (Application Programming Interface) einfach zu erlernen und unabhängig von der Java Version und vom Netzwerk. Durch seine Peer-to-Peer-Architektur ist es besonders gut für selbstständig interagierende, heterogene Agenten geeignet. Jade ist ein opensource Projekt, die Weiterentwicklung wird durch eine breite Gemeinschaft aus Industrie (Motorola, HP, Siemens, etc.) und Universitäten von ca. 1.000 Mitgliedern und einem Governing Board gewährleistet.

Agenten kommunizieren über eine Agent Communication Language (ACL) die einerseits komplexe Konversation gestattet und andererseits durch vorgefertigte Interaktionen, wie Verhandlungen, Auktionen, oder Delegation, den Anwender von komplizierten Implementationen befreit.

\subsection{Implementation der Agenten}

Das beschriebene Simulationsmodell basiert im Wesentlichen auf zwei komplexen Agententypen. Zum einen dem Agent „Börse“, der die Simulation überwacht und steuert und zum andern heterogenen Agenten, die die Investoren repräsentieren.

www.fipa.org 


\subsubsection{Agent „Börse“}

Der Agent „Börse“ (Abbildung 4) übernimmt neben den klassischen Aufgaben einer Börse auch die Initiierung, Steuerung und die Beendigung der Simulation. Er fordert die Agenten zur Abgabe eines Gebotes auf und verarbeitet die eintreffenden Orders innerhalb des Auktionsmechanimus, wie unter 3.4 beschrieben, zu einem Einheitskurs. Der Einheitskurs und das entstandene Volumen werden gesammelt und auf einem Massenspeicher für die späteren statistischen Auswertungen abgelegt. Der Agent „Börse“ generiert außerdem die exogenen Werte Zins und Gewinn je Aktie, die über eine zyklische Funktion mit normalverteilten Abweichungen erzeugt wird. Auch diese Werte werden auf dem Massenspeicher abgelegt. Die Kontrolle übernimmt die Kommunikation und Steuerung der Agenten. Es werden die aktuellen Marktdaten für Preis und Volumen und die fundamentalen Werte für Zins und Gewinn je Aktie den Agenten zur Verfügung gestellt. Außerdem erfolgt das Settlement der getätigten Umsätze indem Aktien und Cash den Agenten zu- bzw. abgebucht werden. Bei Erreichen des letzten Handelstages fordert der Agent „Börse“ die Agenten zur Deregistrierung auf und beendet die Simulation.

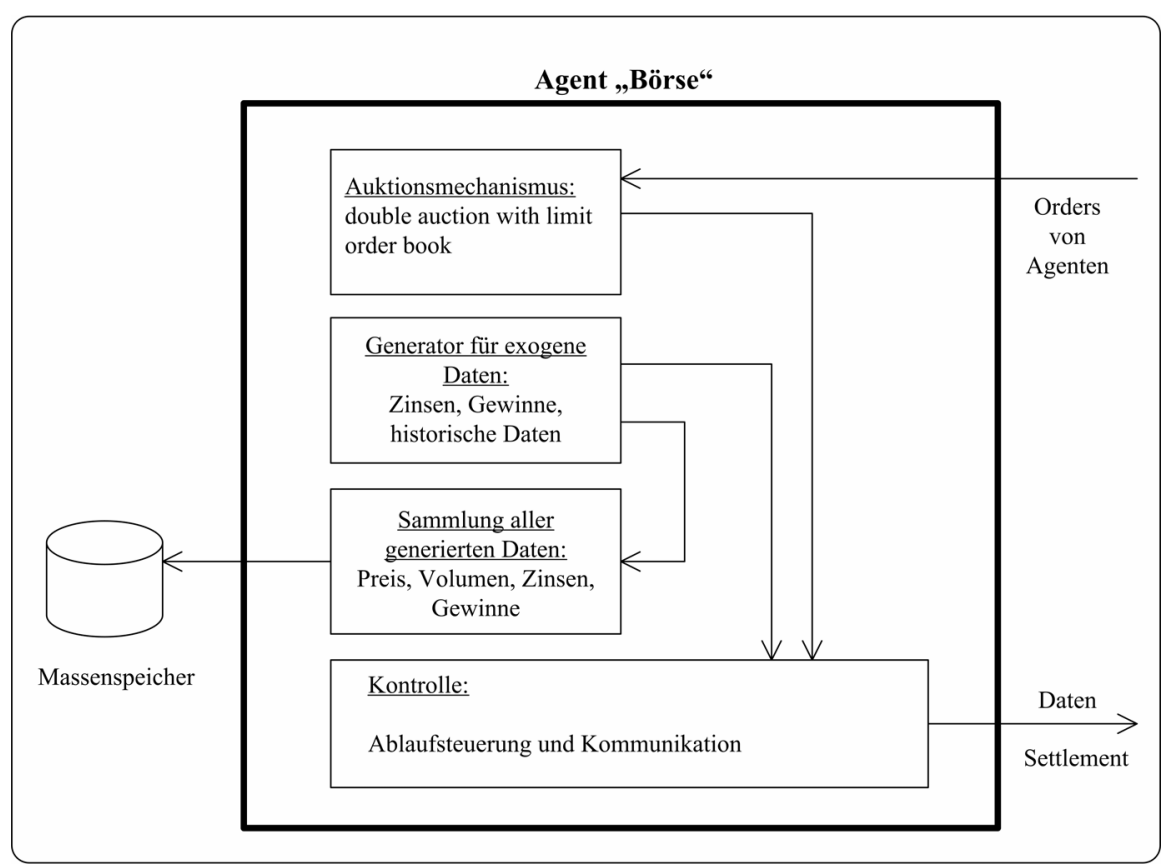

Abbildung 4: Blockdiagramm von Agent „Börse“ 


\subsubsection{Agent ,Investor“6}

Der Agent „Investor“ (Abbildung 5) modelliert die beiden Anlegertypen wie unter 3.1 beschrieben. Er verfügt über eine Nachrichtenschlange, die eingehende Nachrichten für die Weiterverarbeitung zwischenspeichert und veraltete Nachrichten löscht. Das Portfolio zeigt den Bestand an Aktien und Cash an. Die Börse verbucht innerhalb des Portfolios die Käufe oder Verkäufe. Jeder Agent verfügt über einen Regelsatz (fundamentale Regel, Trendregel) mit dem er sein Verhalten optimieren kann. Ein lokaler Datenspeicher zeichnet alle Aktivitäten des Agenten für spätere statistische Auswertungen auf und legt sie auf einem Massenspeicher ab.

Das Verhalten der Investoren wird von einer finite state machine kontrolliert. Die verschiedenen Eingangsparameter wie persönlicher Erfolg, der Erfolg der direkten Nachbarn, der aktuelle Zustand und die aktuell verwendete Regel werden für die Übergangsfunktion verwendet. Außerdem werden für die Übergangsfunktion individuelle Aktivitätsschwellen verwendet, die ein gleichzeitiges Handeln aller Investoren einer Gruppe ausschließen.

Der Wechsel zwischen den Handelsregeln ist ausschlaggebend für die Diffusionsprozesse des Investorenverhaltens innerhalb des Kommunikationsnetzwerkes. Die Warteschlange an eingehenden Nachrichten wird regelmäßig überprüft. Sollte die Mehrheit der direkten Nachbarn erfolgreicher mit einer anderen Handelsstrategie sein und die Erfolgsdifferenz über dem Schwellenwert liegen, so passt der Agent seine Handelsstrategie der erfolgreicheren Mehrheit an.

Für den Aktienkurs $\rho$ und den individuellen Schwellenwert $\tau$, den Zinssatz r, den Gewinn pro Aktie s und die aktuell verwendete Regel $\gamma$ wird eine Limit Order für einen fundamental orientierten Investoren folgendermaßen generiert:

$$
\begin{array}{lrll}
\text { If } & \gamma(\mathrm{r}, \mathrm{s})+\tau>\rho & \rightarrow & \text { buy at limit } \gamma+\tau \\
\text { If } & \gamma(\mathrm{r}, \mathrm{s})-\tau<\rho & \rightarrow & \text { sell at limit } \gamma-\tau
\end{array}
$$

Der Agent kann nur soviel Aktien kaufen bzw. verkaufen wie er über Bargeld, bzw. Aktien in seinem Portfolio verfügt. 


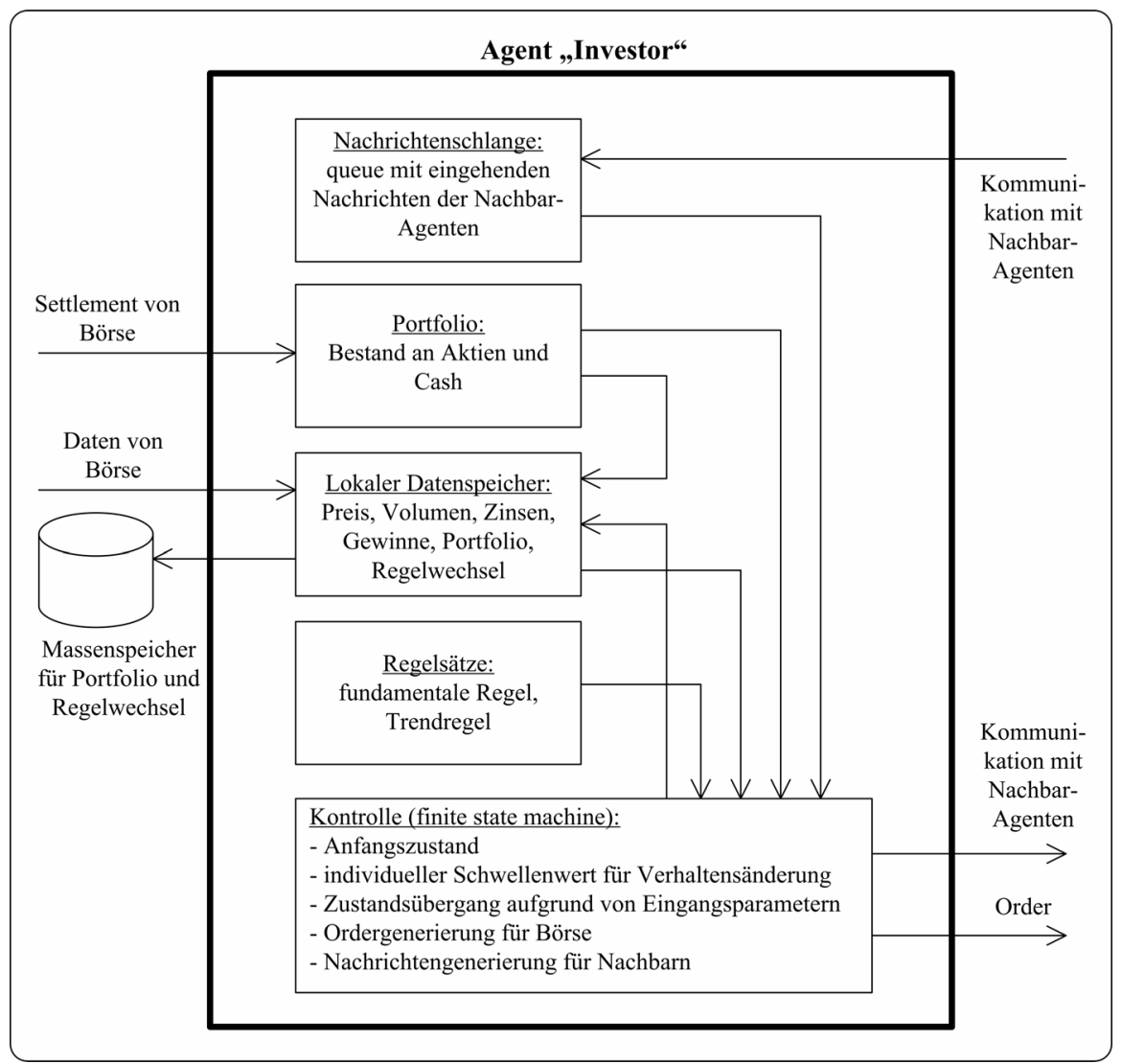

Abbildung 5: Blockdiagramm von Agent „Investor“

\subsection{Implementation der Agentenkommunikation}

Die Inter-Agenten-Kommunikation ist ein wesentlicher Faktor für das adaptive Verhalten der Agenten. Die Kommunikation ist entsprechend der FIPA Agent Communication Language (ACL) definiert worden. Abbildung 6 zeigt zur besseren Visualisierung der Agentenkommunikation ein Agent-UML (AUML) Sequenz Diagram [Ode $\left.{ }^{+} 00\right]$.

Die Börse (,exchange“) generiert zu Beginn die für die Entscheidung der Agenten benötigten historischen Zeitreihen für Zinsen und Gewinne („create history“), wartet bis sich alle Agenten angemeldet haben und fordert zur Abgabe eines Gebotes (,cfp“) in Form von einer Kauf- bzw. Verkaufsorder mit Limit und Stückzahl, oder in Form einer Mitteilung über Inaktivität für den aktuellen Handelstag, auf. 
Alle x Handelstage bewerten die Agenten ihren monetären Erfolg der letzten $\mathrm{x}$ Handelstage und kommen zu einem positiven (bei Gewinn) oder zu einem negativen Urteil (bei Verlust). Sie teilen dieses Urteil ihren unmittelbaren Nachbarn in Form einer Nachricht mit (,send (success, failure)“). Die Nachrichten werden in einer Eingangsschlange gesammelt und dienen als Parameter für spätere eventuelle Verhaltensänderungen. Die Nachrichten verfügen über eine Gültigkeit und werden bei Überschreitung gelöscht.

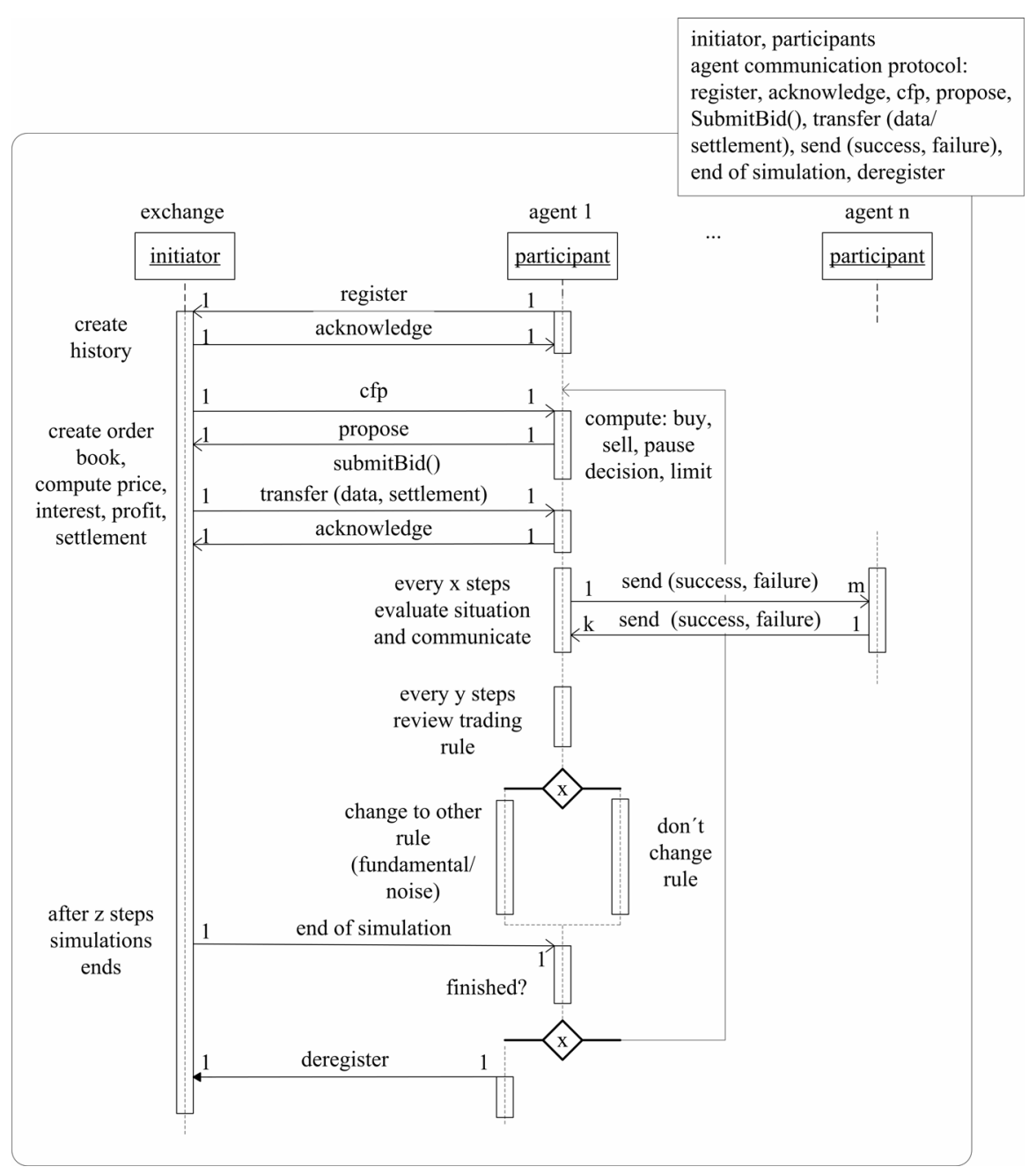

Abbildung 6: AUML Chart des Simulationsmodells 
Alle y Handelstage bewerten die Agenten den Erfolg ihrer Handelsstrategie im Verhältnis zu ihrem monetären Erfolg und dem monetären Erfolg ihrer direkten Nachbarn. Bei unzureichendem eigenem monetärem Erfolg wird die Eingangsschlange von Nachrichten der unmittelbaren Nachbarn untersucht. Sollte sich dort eine Anzahl von positiven Nachrichten von Agenten mit abweichender Handelsstrategie befinden die über einem individuellen Schwellenwert liegt, erfolgt eine Angleichung der eigenen Handelsstrategie an die der erfolgreichen Agenten (,change to other rule, don't change rule“).

Ist der Simulationsverlauf noch nicht beendet beginnt ein neuer Handelstag, ansonsten melden sich die Agenten bei der Börse ab und die Simulation endet (,,deregister").

\section{Testläufe}

Der FASM befindet sich momentan in einer extensiven Testphase. Folgende Input- und Output-Parameter werden untersucht:

\section{Input Parameter:}

- Anzahl der Agenten

- Anzahl der Handelstage

- Initiale Verteilung von fundamentalen und trend-orientierten Investoren

- Initiale Verteilung von Bargeld und Aktien zwischen den Agenten

- Streuungsbreite von individuellen Merkmalen wie Aktivierungsschwellen variierte Handelsstrategien

- Zyklische Funktionen für Zinsen und Gewinn pro Aktie

- Initiale Preishistorie für Trend Investoren

- Agenten Netzwerk Matrix charakterisiert durch durchschnittliche Pfadlänge and clustering coefficient

- Zeitintervalle für die Überprüfung auf Regelwechsel und Kommunikation

\section{Output Parameter:}

- Zeitreihen für den Aktienkurs und Umsatz für die Analyse auf „fat tails“, volatility clustering und Volatilität Volumen Korrelation.

- Tägliches Agenten Portfolio mit der Menge an Aktien und Bargeld, für die Beurteilung des Erfolgs einzelner Agentenportfolios und Handelsstrategien 
- Das Auftreten von Regelwechsel im Verhältnis zu Preisänderungen, individuelle Aktivitätsschwellen für den Nachweis von Herdenverhalten

- Die tägliche räumliche Verteilung von Käufern, Verkäufern und Handelsstrategien innerhalb des Agenten Netzwerkes

\section{Zusammenfassung}

Die Mikrosimulation von Kapitalmärkten kann trotz ihrer recht kurzen Geschichte bereits erste bedeutende Erkenntnisfortschritte vorweisen. Interdependenzen und wechselseitige Dynamiken können in diesen Modellen besser untersucht werden, als mit herkömmlichen analytischen Methoden. Sie ergänzen damit die klassischen Theorien über die Effizienz der Märkte. Die universellen stilisierten Fakten der Kapitalmärkte stellen statistische Phänomene dar, die als empirische Validierung für Marktmodelle dienen können.

Die Implementationen von Marktmodellen ist äußerst heterogen und dadurch kaum nachvollziehbar und vergleichbar. Eine Standardisierung würde Modelle nicht nur vergleichbarer machen, sondern würde auch den Entwicklungsaufwand auf die wesentlichen Details reduzieren. Der FASM stellt die Bemühung dar, durch die standardisierte Middleware Jade, die FIPA konform ist, eine Plattform zur besseren Untersuchung von dynamischen Preisbildungsprozessen bereitzustellen.

Der FASM knüpft dabei an die Tradition des Modellbaus für Kapitalmärkte an. Im Hinblick auf eine realitätsnahe Modellierung der Agenten-Kommunikation und Informationsdiffusion geht dieses Modell jedoch weit über die Vorgängermodelle hinaus. Die Erforschung von sozialen Netzwerken und der Dynamik von Ansteckungsprozessen hat in den letzten Jahren einen Aufschwung erfahren. Der FASM verbindet beide Forschungsbereiche und soll bereits gewonnene Erkenntnisse auf die Verwendbarkeit innerhalb der Erforschung von Preisbildungsprozessen hin überprüfen.

\section{Literatur}

[BaA199] Barabasi, A.-L.; Albert, R.: Emergence of Scaling in Random Networks. Science 286, 1999, S. 509-512.

[BaBo03]Barabasi, A.-L.; Bonabeau, E.: Scale-Free Networks. Scientific American, 2003.

[Bel $\left.{ }^{+} 03\right]$ Bellifemine, F.; Caire, G.; Poggi, A.; Rimassa, G.: Jade, A White Paper. exp 3, 2003, S. 6-19. 
[Bia $\left.{ }^{+} 95\right]$ Biais, B.; Hillion, P.; Spatt, C.: An Empirical Analysis of the Limit Order Book and the Order Flow in the Paris Bourse. The Journal of Finance 50, 1995, S. 16551689.

[Bou 02] Bouchaud, J.-P.; Mezard, M.; Potters, M.: Statistical properties of stock order books: empirical results and models. Quantitative Finance 2, 2002, S. 251-256.

[Con01] Cont, R.: Empirical properties of asset returns: stylized facts and statistical issues. Quantitative Finance 1, 2001, S. 223-236.

[CoBo00] Cont, R.; Bouchaud, J.-P.: Herd behavior and aggregate fluctuations in financial markets. Macroeconomic Dynamics 4, 2000, S. 170-196.

[Con99] Cont, R.; Ecole Polytechnique, P.: Statistical properties of financial time series, 1999.

[Far01] Farmer, J. D.: Toward Agent-Based Models for Investment, Developments in Quantitative Investment Models 2001.

[FaSh02] Farmer, J. D.; Shareen, J.: The price dynamics of common trading strategies. Journal of Economic Behavior and Organization, Vol. 49, No. 2, pp. 149-171, 2002.

[KaTv82] Kahneman, D.; Tversky, A.: Judgment Under Uncertainty. Cambridge University Press: Boston, 1982.

[LeB $\left.{ }^{+} 94\right]$ LeBaron, B.; Holland, J.; Arthur, B. W.: Artificial Economic Life: A Simple Model of a Stockmarket. Physica D 75, 1994, S. 264-274.

[Lev $\left.{ }^{+} 00\right]$ Levy, H.; Levy, M.; Solomon, S.: Microscopic Simulations of Financial Markets. Academic Press: New York, 2000.

[LoM $\left.{ }^{+} 02\right]$ Lo, A. W.; MacKinlay, A. C.; Zhang, J.: Econometric models of limit-order executions. Journal of Financial Economics 65, 2002, S. 31-71.

[Lux00] Lux, T.: Volatility Clustering in Financial Markets: A Microsimulation of Interacting Agents. International Journal of Theoretical and Applied Finance 3, 2000, S. 675702 .

[LuMa99] Lux, T.; Marchesi, M.: Scaling and criticality in a stochastic multi-agent model of a financial market. Nature, Vol. 397, No. 11, pp. 498-500, 1999.

[MaMi01] Maslov, S.; Mills, M.: Price fluctuations from the order book perspective - empirical facts and a simple model. Physica A 299, 2001, S. 234-246.

[Ode $\left.{ }^{+} 00\right]$ Odell, J. H.; Parunak, H. v. D.; Bauer, B.: Extending UML for Agents. Proceedings of the Agent-Oriented Information Systems Workshop at the 17th National Conference on Artificial Intelligence, 2000, S. 3-17.

[PaVe01] Pastor-Satorras, R.; Vespignani, A.: Epidemic Spreading in Scale-Free Networks. Physical Review Letters 86, 2001, S. 3200-3203.

[Pod99] Poddig, T.: Handbuch Kursprognose, Quantitative Methoden im Asset Management. Uhlenbruch Verlag: Bad Soden, 1999. 
[Rab ${ }^{+}$03] Raberto, M.; Cincotti, S.; Dose, C.; Focardi, S. M.; Marchesi, M.; Genova, U. d.: Price formation in an artificial market: limit order book versus matching of supply and demand, 2003.

[Rab $\left.{ }^{+} 01\right]$ Raberto, M.; Cincotti, S.; Focardi, M. S.; Marchesi, M.: Agent-based simulation of a financial market. Physica A 299, 2001, S. 320-328.

[Sim97] Simon, H. A.: Models of Bounded Rationality, Vol. 3: Emperically Grounded Economic Reason. MIT Press: Boston, 1997.

[Smi ${ }^{+}$03] Smith, E.; Farmer, J. D.; Gillemot, L.; Krishnamurthy, S.: Statistical theory of the continous double auction. Arbeitsbericht: Santa Fe Institute: Sante Fe, New Mexico, USA, 2002.

[Spi02] Spiwoks, M.: Vermögensverwaltung und Kapitalmarktprognose - Überprüfung der Prognosekompetenz ausgewählter deutscher Vermögensverwalter. Peter LangEuropäische Hochschulschriften: Frankfurt/M., 2002.

[Spi03] Spiwoks, M.: Qualität der Zinsprognosen deutscher Banken - Eine empirische Analyse. Kredit und Kapital 3, 2003, S. 289-308.

[Spi04] Spiwoks, M.: Die Verwendbarkeit der ZEW-Aktienindex-Prognose für aktive Portfoliomanagement-Strategien. Jahrbücher für Nationalökonomie und Statistik, 2004.

[WaCh03] mWang, F., Xiao; Chen, G.: Complex Networks: Small-World, Scale-Free and Beyond. IEEE Circuits and Systems Magazine 1, 2003, S. 6-20.

[WaSt98] Watts, D. J.; Strogatz, S. H.: Collective dynamics of 'small-world' networks. Nature 393, 1998, S. 440-442. 